POPULATION: We are creating recombinant fusions in which glial cell-derived neurotrophic factor (GDNF) is linked to galectin-3 (G3), a human protein that binds to extracellular beta-galactoside glycans and glycosaminoglycans. GDNF-G3 fusion proteins will circumvent major therapeutic shortcomings of early GDNF human trials by anchoring GDNF to the midbrain in a preclinical animal model of PD over a therapeutically-relevant timescale in order to achieve DA neuron rescue. Further, in PD patients, we have detected significantly dysregulated dopamine signaling in peripheral, blood-derived monocytes, suggesting a systemic dopamine signaling change in PD. RESULTS/ANTICIPATED RESULTS: Based on results from published human NTF administration trials, we anticipate that a successful intervention using GDNF-G3 will result in rescue or delayed degeneration of midbrain dopaminergic neurons in a murine PD model. Outcome measures include behavioral PD phenotype testing via rotarod and pole descent compared to non-parkinsonian control animals, as well as corroborating immunohistological evidence from immunohistochemical examination of post-mortem brain tissue from the same animals to examine degree of degeneration. DISCUSSION/SIGNIFICANCE OF IMPACT: Current treatments for PD, whether pharmacological or surgical, center on alleviating movement symptoms that impair daily function - in other words, largely palliative care. Little has been accomplished by way of rescue of dopaminergic neurons or slowing disease progression using standard-of-care therapy. If successful, GDNF-G3 constructs administered intracranially at the site of degeneration would represent a milestone on the path to treating the basic pathology associated with PD, while addressing major shortcomings in earlier NTF-delivery attempts, namely NTF diffusion away from target site.

3369

Assessment to Action: Engaging network member's in identifying needs and directions of network improvement

Paul Estabrooks ${ }^{1}$, LaKaija Johnson, Jolene Rohde, Carol Geary, Lani Zimmerman, Matthew Rizzo, MD, FAAN, FANA and Mary Cramer ${ }^{1}$ University of Nebraska Medical Center

OBJECTIVES/SPECIFIC AIMS: To complete a needs assessment and action planning process that engaged clinical and translational research network members in identifying needs through survey feedback, characterizing the needs in small group sessions, and developing recommendations for action at the network's annual scientific meeting. METHODS/STUDY POPULATION: The project included (1) a survey of 357 members across partner institutions from the Great Plains IDeA CTR Network, (2) 6 - 90 minute brainstorming sessions to characterize needs identified through survey assessment, and (3) 6 - 60 minute sessions to develop recommendations for network improvement based on the characterization activity. Approximately 75 members participated in the characterization and recommendation sessions. RESULTS/ANTICIPATED RESULTS: Seven areas of need from the survey were identified based upon the frequency of identification by network members (support to move research across the translational spectrum, database design and management, data access and sharing, data analysis, recruitment and retention of subjects, support for members who have submitted grants but were repeatedly unsuccessful, mentoring). Members indicated which characterization sessions they were interested in attending and based on the enrollment numbers needs related to unsuccessful grant submitters and mentoring were combined as were needs related to database design and data access-sharing. Sessions resulted in 8 inter-related recommendations for network action that included to (1) develop GP-CTR directory/registry of clinicians, researchers, system partners, that can be used to identify people that want to be involved in research partnerships or mentoring, (2) create a GP CTR Navigators Program to will provide support to network members throughout the collaborative research and grant preparation process, (3) identify and disseminate information about assets (funding, databases/registries) that exist amongst network partners that can be leveraged by member, (4) develop a searchable repository of evidence-based interventions for T3/T4 efforts, (5) review GP CTR supported professional development, and technological resource offerings and identify potential gaps, (6) facilitate opportunities for peer support/networking, (7) provide guidance to GP CTR network institutions looking to adopt policies that will support translational research collaboration, and (8) identify potential barriers to GP CTR network engagement (i.e., infrastructure, communication, marketing). DISCUSSION/SIGNIFICANCE OF IMPACT: This process allowed for a wide range of network members to contribute to actionable recommendations for CTR leadership to move into action and improve the scientific network's ability to conduct clinical and translational research.

3203

\section{Collaboration in Reappointment, Promotion, and Tenure Guidelines}

Jacqueline Knapke, $\mathrm{PhD}^{1}$, John R. Kues², Stephanie M. Schuckman² and Rebecca C. Lee

${ }^{1}$ Cincinnati Children's Hospital Medical Center and ${ }^{2}$ University of Cincinnati

OBJECTIVES/SPECIFIC AIMS: As the issues facing our global society become more complex, university faculty are called upon to address these contemporary problems using interdisciplinary approaches. But do reappointment, promotion, and tenure (RPT) guidelines reflect and reward this fundamental change in the nature of higher education and scholarly inquiry? After collecting all of the RPT guidelines across the university, our research team at the University of Cincinnati (UC) conducted a content analysis of these documents to determine how collaborative work is defined, interpreted, and supported. In addition, we also sought to identify differences in how collaborative work is valued across disciplines and how that value has changed over time. METHODS/STUDY POPULATION: An initial database was assembled that included two distinct data samples: historical and current. Both included RPT criteria for over 100 disciplinary units at the university. Working with the initial comprehensive database, the team narrowed content by selecting all language related to collaborative work using several relevant keywords or keyword fragments (team, collaborat $\left.{ }^{*}\right]$, disciplin $\left.{ }^{*}\right]$, and interprofessional). This process resulted in a subset of data reflecting the area of interest that could then be coded. Three investigators independently coded common portions of the data for categories. The investigators met regularly to compare the results of their coding, and discrepancies between the investigators' coding schemes were resolved through discussion. The final, common coding scheme will used to code the remainder of the data by each independent investigator. The team meets weekly to discuss significant passages and assign codes, and then reach consensus related to important themes that are identified. Specifically, we will examine the frequency with which collaborative activities are included, the value and emphasis given to them, and the differences across units. Having a historical sample and a current sample also allows us to 